\title{
PAZ Y GUERRA: UN ANÁLISIS A LOS SPOTS POLÍTICO-ELECTORALES TELEVISIVOS DE LA CAMPAÑA AL SENADO DE COLOMBIA DESDE LA TEORÍA DE LA RELEVANCIA
}

\author{
Yeny Alexandra Pulido Aguirre \\ Universidad El Bosque (Colombia) / Universidad Nacional de Educación a Distancia (España) \\ ypulidoa@unbosque.edu.co; ypulido5@alumno.uned.es
}

Recibido: 14/02/2020 - Aprobado: 02/04/2020

DOI: doi.org/10.17533/udea.lyl.n78a10

\begin{abstract}
Resumen: Se identificaron los sentidos explícitos e implícitos sobre el conflicto armado en spots político-electorales de la campaña legislativa de 2014. Veinte spots fueron analizados según la teoría de la relevancia de Sperber y Wilson. Se encontró que la conceptualización de la paz y la guerra se dio casi exclusivamente de manera implícita y que el tema más tratado fue el de la paz, el cual generó polarización política ante las negociaciones de paz del Gobierno con las FARC. Este discurso, como un rasgo propio de la comunicación, sirve también para la comprensión del mensaje del candidato por sus electores.
\end{abstract}

Palabras clave: teoría de la relevancia; implicaturas; spot político-electoral; elecciones al Congreso; Colombia.

\section{PEACE AND WAR: AN ANALYSIS OF THE POLITICAL-ELECTORAL TELEVISION SPOTS OF CONGRESS OF COLOMBIA ELECTION CAMPAIGN FROM THE RELEVANCE THEORY}

\begin{abstract}
The explicit and implicit meanings about the armed conflict were identified in political-electoral spots of the 2014 Senate campaign. Twenty spots were analyzed according to the theory of relevance of Sperber and Wilson. It was found that the conceptualization of peace and war occurred almost exclusively implicitly and that the most discussed issue was that of peace, which generated political polarization in the face of the Government's peace negotiations with the FARC guerrilla. This discourse, as a characteristic of communication, also serves for the understanding of the candidate's message by his voters.
\end{abstract}

Key words: relevance theory; implicatures; political-electoral spot; Senate elections; Colombia. 


\section{Introducción}

$\mathrm{E}$

n el 2014 se desarrolló la campaña electoral para Senado de la República de Colombia, influenciada por las negociaciones de paz entre el Gobierno Nacional y la guerrilla de las FARC. Se trataba de un momento histórico, estaba en juego el futuro del país, terminar o no el conflicto armado interno de más de 50 años. Dado que se trataba de una elección a nivel nacional, como de costumbre, los candidatos usaron spots televisivos con el fin de llegar a todo el país. El spot político-electoral, según Contreras (2006), se constituye en una narración que permite conformar un imaginario social colectivo dominante, porque esta narración exhibe una fusión de imaginarios políticos de los electores y de los candidatos, con una mezcla de los valores deseados por los electores y los que posee el candidato. De esta idea, cabe resaltar que el candidato es quien domina el contenido del mensaje y, así mismo, quien puede manipularlo si así lo desea.

Esto no es una suspicacia, la teoría de la relevancia, de Wilson y Sperber, advierte que el comunicador construye sus enunciados bajo los límites de sus propias capacidades y preferencias, con el objetivo de ser comprendido (2005, pp. 230-231; 2012, p. 65). Además, el esfuerzo por ser comprendido, también, supone la creación de un mensaje relevante, es decir, uno que se conecte con la información previa disponible para producir efectos cognitivos, conclusiones que importan a un individuo: confirmación, revisión o abandono de supuestos previos, fortalecimiento de una suposición existente, contradicción y eliminación de una suposición existente o combinación con una suposición existente para producir implicaciones contextuales (Wilson \& Sperber, 2005, p. 223; Wilson \& Sperber, 2012, p. 102; Wilson \& Matsui, 2012, p. 200). Esta es la razón que motiva al oyente hacia la interpretación del sentido del hablante, pues un efecto cognitivo positivo es una diferencia ventajosa en la representación del mundo del individuo: una conclusión verdadera, por ejemplo, las conclusiones falsas no son ventajosas (Sperber \& Wilson, 1995, citados por Wilson \& Sperber, 2005).

Lo anterior sugiere que un candidato, en el caso del spot político-electoral, debe informarse del contexto cognitivo de sus votantes, el cual podría consistir en sus necesidades más apremiantes y la agenda pública del momento, que daría como resultado el marco conceptual del votante. De ahí la importancia de que el candidato realice un estudio en el que identifique y 
jerarquice «los problemas, necesidades, frustraciones y expectativas de la sociedad, así como los candidatos con quienes mejor se relaciona la posible solución de los mismos» (De Aragón, 2011, p. 66). Esto con el fin de garantizar su adecuación al electorado (ODCA, 2006, p. 69), ya que la audiencia presta atención a lo que le interesa o a lo que concuerda con lo que piensa (García Beaudoux, D'Adamo, \& Slavinsky, 2005, p. 184).

El asunto no termina allí, pues también se ha de tener en cuenta que, según Wilson y Sperber (1998a, citado en Wilson \& Sperber, 2012, p. 71), todo mensaje es una codificación lingüística de lo que el hablante pretende comunicar, y solo es una indicación esquemática del sentido del hablante, es decir, lo explícito. Esto significa que, el hablante no dice todo lo que quiere decir con las palabras que ha elegido para la construcción del enunciado, sino que ese enunciado evoca ideas más allá de lo codificado lingüísticamente. De este modo, el enunciado evoca un conocimiento conceptual del contexto cognitivo compartido y produce inferencias, implicaturas, que permiten la construcción del sentido del hablante. Estas implicaturas son la información implícita que ofrece el enunciado.

\subsection{El porqué de este estudio}

Esta pregunta se responde partiendo del hecho de que hay un mayor interés por el estudio de los spots político-electorales televisivos para la Presidencia, sobre todo de aquellos más populares; incluso de otras piezas publicitarias.

Uribe y Rincón (2016) estudiaron los spots televisivos de la campaña a Senado y Presidencia de 2014, aquellos de mayor resonancia y difusión, a través del modelo de Beaudoux, D’Adamo y Slavinsky (2005) y los criterios de story-telling, story-living, branding e issues. Los autores encontraron que se dio una confrontación entre dos modos de concebir el país, una que apeló a un futuro de paz y más de lo mismo de Santos y, la otra, a un futuro-pasado que consiste en volver a los tiempos de Uribe como acto de restauración moral; no obstante, dejan de lado los discursos menos populares, de sectores marginados que no cuentan con recursos económicos que les permita hacer más visibles sus piezas publicitarias.

Otros autores tuvieron hallazgos semenjates, solo que en la campaña a la Presidencia, como Rivas y Roll (2008) en los spots televisivos de la campaña a la presidencia, a través de la técnica 
de análisis de contenido y de la conceptualización de la agenda política. También se dio el caso de Hernández Bayter (2015), en los debates para primera y segunda vuelta. Este autor analizó los mecanismos lingüístico-discursivos empleados por los candidatos a la Presidencia de Colombia 2014-2018, Juan Manuel Santos (JMS) y Oscar Iván Zuluaga (OIZ), durante el debate entre la primera y la segunda vuelta. A través de una metodología lexicométrica, el autor observó que el uso del artículo determinado e indeterminado permite vehicular una estrategia discursiva en el tema de la paz, que también adquiere las características de paz determinada o indeterminada, la primera impulsada por JMS como el único líder que la puede conseguir, la segunda, propuesta por OIZ y que corresponde a una nueva forma de conseguirla.

Richard (2015) hizo su estudio en diferentes piezas publicitarias. La autora consideró que una campaña electoral tiene una estructura narrativa semejante a una obra de teatro. La autora identificó toda la estructura actancial de las narrativas que promovieron JMS y OIZ en la primera y segunda vuelta para la Presidencia de Colombia. El tema de la paz fue impuesto por JMS sin dificultad, y recuperado tardiamente por su principal contendor, OIZ. Los dos candidatos trataron este tema «en un tipo de narración específico, el de la búsqueda, en el cual la política se asimila a una lucha entre varios personajes para la consagración de un ideal encarnado por el héroe» (Richard, 2015, § 3).

Desde otra perspectiva, en cuanto a la idenficación de temas, obviando las interpretaciones del sentido de los mensajes, el macroproyecto de la MOE (2015) reunió a académicos de diferentes regiones del país para estudiar el cubrimiento de la campaña electoral en los medios de comunicación de sus regiones. Se encontró que la paz fue un tema importante en las campañas para el Congreso y la Presidencia de la República en diferentes medios de comunicación nacionales y locales.

Evidentemente, se observa un mayor interés por el estudio de la comunicación políticoelectoral de la campaña para la Presidencia, pese a que las elecciones legislativas son igualmente importantes, pues se trata de los cargos públicos más poderosos en un país. No obstante, el hecho que sobresale en la defensa del estudio de la publicidad para el Congreso en Colombia se relaciona con el hecho de que la campaña legislativa se desarrolló previamente a la presidencial, convirtiéndola en un escenario de prueba y de batalla de las ideas que se pretendían desarrollar en la campaña a la Presidencia. 
Paralelamente, los estudios anteriormente referenciados no explican cómo llegaron a la interpretación de esos spots y las otras piezas de comunicación. No se sabe qué fue comunicado explícitamente y qué implícitamente, qué modo de comunicación prefiere el candidato (explícito o implícito). Estas características propias de la comunicación inducen diversas preguntas sobre las ideas comunicadas por los partidos políticos que participaron en la contienda electoral de 2014 al Congreso: ¿Cuál es la idea de paz y guerra que se transmiten en estos spots? ¿Se expresa de manera explícita o implícita? ¿Cuál es la idea de paz y guerra de cada partido político? Así, el propósito de este trabajo consistió en identificar los sentidos explícitos e implícitos sobre la paz y la guerra que los partidos políticos ponen en los spots mencionados; en este artículo se presentan los resultados parciales de un proyecto más amplio, como parte de una tesis doctoral, que incluye el estudio de spots para la Presidencia en ese mismo año y en el que se examina la situación de Colombia expresada en los mismos.

Para cumplir con este propósito, los spots se observaron a través de la teoría de la relevancia de Wilson y Sperber (2005; 2012), ya que esta trata la identificación del contenido explícito tanto como el inferencial, lo que permite al oyente la construcción de una hipótesis sobre el significado del hablante.

\subsection{Colombia y su lucha por la paz}

Los spots analizados se difundieron en un momento en que el principal tema de discusión del momento eran los diálogos de paz del Gobierno Nacional con las FARC-EP (Fuerzas Armadas Revolucionarias de Colombia-Ejército del Pueblo). Esos diálogos se remontan al año 2012, cuando el gobierno del presidente JMS negociaba la paz con las FARC en La Habana, (Cuba). El proceso tuvo como propósito dar fin a más de 50 años de confrontación armada. Esta negociación se desarrollaba en secreto hasta que Álvaro Uribe (AU), expresidente de Colombia y contradictor del gobierno de JMS, reveló su existencia. Entonces, JMS se vio obligado a confirmarlo ante los colombianos. A partir de ese momento, AU reiteró sus argumentos en contra de dicha negociación. Por un lado, el supuesto de que los guerrilleros serían indultados, en consecuencia, no irían a la cárcel por los crímenes que cometieron. De otro lado, la idea de que a los guerrilleros se les daría participación en la política nacional, a través de curules en el Congreso de la 
Republica. Este supuesto se basa en el hecho de que, en el pasado, cuando el grupo guerrillero M19 se desmovilizó, sus miembros recibieron indulto, además de que ingresaron a la vida política del país, logrando ocupar cargos de elección popular.

Estos argumentos pretendían mostrar que una negociación, como la que se dio en La Habana, podría conducir a la injusticia de perdonar a los guerrilleros por los crímenes cometidos y, en cambio, premiarlos con el otorgamiento de cargos públicos. La cuestión es que, durante el conflicto armado, las FARC ejercieron dominio sobre varias regiones del país — aprovechando la poca o nula presencia del Estado-, a través de la confrontación armada contra las fuerzas policiales y del ejército, del cobro de impuestos de guerra a los campesinos (dinero o posesiones como ganado, comida, etc.), reclutamiento forzado de niños y adultos, desplazamiento forzado de personas, asesinato de opositores, intimidación a las comunidades, etc. En cifras, «ocho millones de víctimas, entre los siete millones de desplazados, más de 26000 muertos, decenas de miles de desaparecidos» (Lafuente, Javier; Palomino, Sally; Marcos \& Ana, 2016).

En el 2014, los diálogos continuaron, con indicios de que podrían tener éxito. Fue así como las fuerzas políticas tomaron partido frente al tema para capitalizarlas en las elecciones para el Congreso de la República. El Partido Liberal Colombiano (PLC) y el Polo Democrático Alternativo (PDA) apoyaron públicamente la continuación de la negociación. Del PLC se destaca como candidato una víctima directa del conflicto, el profesor Gustavo Moncayo. Este profesor es padre del soldado Pablo Emilio Moncayo, quien fue secuestrado por las FARC el 21 de diciembre de 1997. El profesor Moncayo colgó cadenas sobre su cuerpo durante años, caminó más de 3000 kilómetros y viajó a 14 países «para reclamar un intercambio de secuestrados por guerrilleros presos y un acuerdo de paz en Colombia» (El Mundo, 2010, § 4). Finalmente, luego de años de manifestaciones, el militar fue liberado tras 12 años de secuestro. A partir de esta experiencia, Gustavo Moncayo sentenció que «hay que cambiar toda esa "historia trágica" de los colombianos» (El Mundo, 2010, § 13) por lo cual estaba de acuerdo con el proceso de paz.

Del otro lado, se encontraba el expresidente AU y su partido Centro Democrático (CD), junto al Partido Conservador Colombiano (PCC). Estas fuerzas políticas continuaron con los supuestos de que los guerrilleros no irían a la cárcel y que recibirían curules en el Congreso. La estrategia se vio fortalecida con la participación de AU como líder de una lista cerrada para el Senado, dándole un mayor perfil a su partido (Pachón, 2014, p. 184). 
Finalmente, después de todo, los resultados de la votación para el Senado del 9 de marzo de 2014 fueron los siguientes: el Partido de la "U" obtuvo el $15.58 \%$ de las curules; el PLC, el $12.22 \%$; el PDA, el $3.78 \%$; mientras que, por otro lado, el CD consiguió el $14.29 \%$ de las curules y el PCC, el 13.58 \% (Colombia.com, 2014). Así las cosas, continuó la campaña para la presidencia, cuya primera vuelta sería el 25 de mayo. Al no definirse el presidente, no fue sino hasta el 15 de junio que los colombianos prefirieron finalmente elegir el proyecto que garantizaría la continuidad de los diálogos de paz: el de JMS.

\section{Metodología}

Este es un estudio mixto, cuya parte cuantitativa se limita a cuantificar enunciados e implicaturas para dar un panorama general sobre cuál fue el tema más tratado. En cuanto a lo cualitativo, describe un fenómeno que ocurrió naturalmente para tratar de descubrir sus patrones de comportamiento. Esto es, se describe el sentido de enunciados relacionados con el tema de la paz y la guerra, a través del modelo teórico y metodológico de la teoría de la relevancia, pues permite visualizar el sentido explícito e implícito de los enunciados.

\subsection{Corpus}

La muestra seleccionada es de tipo no probabilística, ya que «la elección de las unidades de análisis no depende de la probabilidad, sino de causas relacionadas con las características de la investigación» (Vilches, del Río, Simelio, Soler, \& Velázquez, 2011, p. 88). Se seleccionaron 20 spots político-electorales televisivos de la campaña electoral para el Senado de 2014 (ver Tabla 1), que se caracterizan por hacer mención sobre la situación de Colombia.

\subsection{Recogida del corpus}

La elaboración del corpus se realizó entre los años 2014 y 2015, mediante los buscadores de internet Google y YouTube, empleando como comando de búsqueda el nombre de los partidos políticos más populares que participaron en la campaña al Senado de 2014: PLC, PCC, PDA, CD 
y CR; como resultado del rastreo, se hallaron los spots disponibles en YouTube, desde donde se mostraban enlaces adicionales asociados a la búsqueda: los spots de otros candidatos y partidos de la misma contienda electoral. Enseguida, se seleccionaron los spots que impulsaran a un candidato o a un partido al Senado en la contienda de 2014, trataran el tema de la paz o la guerra, tuvieran una duración menor a 60 segundos y representaran la totalidad de partidos participantes en la campaña electoral.

\section{Partido}

Código y nombre del spot

Partido Liberal Colombiano
(PLC)
$\begin{aligned} & \text { B. Horacio Serpa Senado } \\ & \text { C. Vota partido Liberal Colombiano, Para que } \\ & \text { vivas Mejor } \\ & \text { D. La paz para que vivas mejor }\end{aligned}$

Partido

Colombiano (PCC)

Cambio Radical (CR)
Movimiento
LINGÜÍSTICA Y LITERATURA
ISSN 0120-5587
E-ISSN 2422-3174
N. ${ }^{\circ} 78,2020242-268$

Autoridades

Indígenas de Colombia - AICO
F. Comerciales polémicos de campañas políticas

Luis Emilio Sierra

G. El Buen Camino con Jorge Hernando Pedraza

H. Comercial Partido Conservador Colombiano

I. Comerciales Polémicos de Campañas Políticas Nora García Burgos Agro Colombiano

J. Cambio Radical No 5 Senado Dusan Vélez.

K. Carlos Fernando Galán habla sobre el Congreso

L. TAITA Luis Humberto Cuaspud al Senado Marque 202 AICO 
Partido Polo Democrático M. Alexander López Maya candidato al Senado Alternativo (PDA) Polo 2

N. Iván Cepeda Senado 10

O. Alberto Castillo Candidato al Senado 2014 2018

P. Dr. Germán Fernández Candidato al Senado Polo 16

\section{Centro Democrático (CD)}

\section{Organización de los Pueblos \\ Indígenas de la Amazonia \\ Colombiana - OPIAC}

Q. Dr. Germán Fernández Candidato al Senado Polo 16

R. Alfredo Rangel Nuestra visión de Paz Centro Democrático

S. María del Rosario Guerra ¿Por qué los candidatos del CD quieren llegar al Congreso

T. Campaña Ati Quigua Senado 2014

\section{Total 20 spots}

Tabla 1. Número de spots estudiados por partido político

\subsection{Diseño de la investigación}

Considerando que en este estudio se analizan textos audiovisuales, se tomó como referencia las fases de análisis del film de Casetti y di Chio (2007, pp. 32-33), en las que se integra el proceso global de comprensión de la teoría de la relevancia y otros elementos metodologicos que 
contribuyen a la organización de los datos del estudio. Estas fases son solo un marco general, donde el proceso global de comprensión tiene protagonismo. A continuación se describe cada una de los pasos:

- Segmentar: Consiste en «la subdivisión del objeto en sus distintas partes. Se trata de individuar en una especie de continuo los fragmentos que lo componen, y de reconocer como algo lineal la existencia de una serie de confines» (2007, p. 32). En esta investigación se trata de la trascripción del mensaje del spot, escena a escena, diferenciando lo expresado a través del medio visual de aquello dicho a través del audio (ver Tabla 2).

\begin{tabular}{cc}
\hline Imagen & Audio \\
\hline & Escena 1 \\
\hline
\end{tabular}

\section{Escena 2}

Tabla 2. Matriz para la trascripción del spot. Fuente: García Beaudoux et al. (2005).

—Estratificar: Se trata de «la indagación "transversal" de las partes individuales, en el examen de sus componentes internos» (p. 32). Esto se da en dos etapas en esta investigación. La primera consiste en tomar de la trascripción los enunciados relacionados, que de alguna manera tratan el tema de la paz o de la guerra. En la segunda etapa se aplica el proceso global de comprensión propuesto por la teoría de la relevancia, el cual, empíricamente, es un proceso online porque la hipótesis sobre las explicaturas, las premisas implicadas y las conclusiones implicadas se desenvuelven en paralelo a partir de un antecedente de expectativas que pueden ser revisadas o elaboradas a medida que el enunciado se desdobla. Si bien esas tareas se desarrollan 
en paralelo, los autores sugieren su descripción de manera secuencial con el objeto de entender cómo funciona la comprensión de los enunciados. Pons (2004) las describió así:

a) Construcción de una hipótesis apropiada sobre el contenido explícito (explicatura) por medio de algunas o todas las siguientes operaciones, aplicadas sólo cuando es necesario para la comprensión del enunciado. En ese sentido, Pons (2004, p. 53) las expone así:

-Desambiguación: sirve para seleccionar una de las acepciones de un término polisémico. Por ejemplo, la descodificación de:

(1) El hombre acababa de terminar la mano, pero estaba húmeda aún.

Debería seleccionar entre una acepción de mano como «parte del cuerpo» o mano como «pasada de pintura».

-Asignación de referente: en el ejemplo anterior, este proceso identificaría el referente correspondiente al sintagma nominal el hombre. Si el que acaba de terminar la mano resulta ser un pintor, seguramente se escogerá la segunda acepción de mano. Si el que la acaba de terminar es un técnico cinematográfico, es muy probable que la acepción seleccionada sea la primera.

-Enriquecimiento: las dos operaciones anteriores no son suficientes para interpretar el enunciado (1) de forma no ambigua. El proceso de enriquecimiento cumple varias funciones; si se centra la atención en dos de ellas, hay que situar primero el enunciado (1) en un lugar y un tiempo determinado. En segundo lugar, hay que precisar el significado de algunas palabras como el adverbio aún. ¿Cuánto tiempo indica aún? Depende de la acepción de mano elegida. Partiendo de la suposición de que se ha escogido la primera de las acepciones y si se sabe que la mano artificial está húmeda aún. ¿Qué quiere decir esto? ¿Qué está húmeda para la escena del pantano? ¿Qué está húmeda para pegársela al actor al que se la van a cortar? ¿Qué se va a deshacer en cuanto se toque? Estas y otras indeterminaciones se resuelven en este punto.

Otros procedimientos de enriquecimiento pragmático: cualquier otro mecanismo que contribuya a delimitar el significado de una palabra, frase o enunciado.

b) Construcción de una hipótesis apropiada sobre los supuestos contextuales pretendidos, cuya función es de premisas implicadas, es decir, una hipótesis que se constituyen en premisa de un razonamiento. 
c) Construcción de una hipótesis apropiada sobre las implicaciones contextuales pretendidas, con la función de conclusión implicada, esto es, varias hipótesis que se constituyen en la conclusión de un razonamiento.

Para emular una situación de comunicación real, se procuró no informarse sobre los sucesos acontecidos en el 2014, por lo que solo estuvo expuesta a las noticias y debates, como cualquier otro ciudadano. Lo anterior con el fin de hallar las implicaturas que un usuario medianamente informado - como gran parte de los colombianos - identificaría en los spots político-electorales televisivos. Así, se limitó a tomar las primeras inferencias que llegaran a la mente, pues así es como se da la interpretación durante el proceso de comunicación, lo cual exigió un esfuerzo para no profundizar en los sentidos de los enunciados.

-Enumerar y ordenar. Es la recensión sumaria de los elementos observados en la que se describen «las correspondencias, las regularidades y los principios que rigen el objeto analizado» (Casetti \& di Chio, 2007, p. 33). Para ello, en este trabajo, se crea una unidad hermenéutica en el programa Atlas-ti, que reúne el material de análisis logrado en la etapa anterior. Ese material se codifica dando cuenta de si el texto es una trascripción - lo expresado explícitamente- o implicaturas (inferencias) y nombre del partido político. Luego, se seleccionan las citas sobre la situación de Colombia para codificarlas en temas, a través de la herramienta de códigos. Enseguida, esos códigos son agrupados en superfamilias las cuales dan cuenta de los temas descubiertos en este análisis.

La nomenclatura de los temas se inspira en la clasificación propuesta por Rivas y Roll (2016, pp. 377-378), de quienes se tomó la denominación de las siguientes categorías (temas): paz, infraestructura, educación, salud, empleo, vivienda, campo, corrupción, temas sociales y otros. A esta lista se le agrega la guerra, que en Rivas y Roll se incluye en «seguridad». En este estudio es importante tener el tema «guerra» como una categoría independiente, debido a que la campaña a Senado discute el tema de paz en oposición a la paz. De otro lado, el tema «empleo» de Rivas y Roll, se incluye en el de «temas sociales» porque tuvo muy pocas menciones en el corpus.

De Rivas y Roll (2016, p. 379) también se consideran las normas y reglas para el análisis. Las normas son las siguientes: 1 . Una cita puede tratar más de un tema. 2. Un spot puede tratar más de un tema. 3. Toda cita (o código) que no corresponda a ningún tema de la lista se clasifica en la 
categoría de "otros"; al respecto, se deben analizar todos los spots seleccionados para este estudio, además de señalarse el tema en cuantas citas aparezcan.

—Recomponer y modelizar: En este punto, «se reconstruye el cuadro global que establece los sentidos, a través de una representación sintética de sus principios de construcción y de funcionamiento» (Casetti \& di Chio, 2007, p. 33). En esta etapa, se toman los informes de Atlasti que reúnen las citas por temas a través de matrices y compilados de información:

a) Tablas de frecuencia de los enunciados explícitos e implícitos.

b) Informe de citas por temas de campaña.

Las tablas se usaron para descubrir los temas más recurrentes y su modo de tratamiento (explícito e implícito). El informe, por su parte, permitió rescatar las citas de los temas de campaña en su forma explícita e implícita.

\section{Resultados}

El tema de la paz se conceptualiza, principalmente, de manera implícita, frente a una referencia explícita sobre la paz (ver Tabla 3). La guerra, por su parte, también se encuentra planteada implícitamente. Ambos temas se observan juntos en algunos spots, pero el de guerra nunca se disocia de la paz. Para el análisis de los enunciados, primero se desambiguan los términos más usados en los spots; segundo, los resultados del análisis se presentan considerando primero los enunciados relacionados con el apoyo a la paz y luego los referidos a la oposición. Estos resultados se dan partiendo del enunciado objeto de análisis, es decir, el contenido explícito. Luego, su análisis, donde se interpreta a partir de las operaciones de construcción de hipótesis, cuando es necesario, y las implicaturas que se derivan del enunciado; la clave para reconocer una implicatura es que se le introduce a partir de los verbos inferir, implicar, sugerir, insinuar y suponer. 


\begin{tabular}{cccc}
\hline & Explícito & Implicaturas & Total \\
\hline Paz & 1 & 32 & 33 \\
Guerra & 0 & 17 & 17 \\
\hline Totales & $\mathbf{1}$ & $\mathbf{4 9}$ & $\mathbf{5 0}$
\end{tabular}

Tabla 3. Relación de las menciones e inferencias sobre la paz y guerra en los spots

Los términos que priman en los spots son paz, guerra y conflicto. [PAZ], se adecua al sentido 3 de la RAE: «f. Acuerdo alcanzado entre naciones por el que se pone fin a una guerra»; no obstante, en el caso del enunciado objeto de este análisis, el fin de la guerra no se da entre dos naciones, sino entre el Estado colombiano y la guerrilla de las FARC. Entonces, el enunciado corresponde a $\left[\mathrm{PAZ}_{2}\right]$ : «f. Acuerdo alcanzado entre dos grupos por el que se pone fin a una guerra», y esos dos grupos son el Gobierno Nacional y la guerrilla de las FARC.

[GUERRA], por su parte, se acerca más al contexto colombiano, y corresponde al sentido 2 de la RAE: [GUERRA $2=$ f. Lucha armada entre dos o más naciones o entre bandos de una misma nación], pero delimitado al sentido ad hoc para este estudio, [GUERRA $3=$ f. lucha armada entre dos bandos de una nación] que para el caso hace referencia al Gobierno Nacional con el grupo guerrillero de las FARC.

Finalmente, [CONFLICTO] se adecua al sentido 2 de la RAE: «Enfrentamiento armado».

De otro lado, a manera de enriquecimiento pragmático, el tema de la paz fue motivado por las negociaciones de paz que desarrollaba el Gobierno Nacional con la guerrilla de las FARC, hecho apoyado por el PLC y el PDA, desaprobado por el CD y el PCC, e ignorado por los partidos CR, OPIAC y AICO. A continuación, se procederá al análisis de los enunciados elegidos:

PLC, spot B: (2) Colombia vive un momento muy importante para la paz. Más guerra, mamola.

Este enunciado implica que Colombia no ha tenido la oportunidad de la paz desde hace mucho tiempo, lo que sugiere a su vez que el presidente está negociando un acuerdo de paz con la guerrilla de las FARC — esta última se denominará Implicatura general 1 en adelante—, además 
de sugerir que hubo otras negociaciones de ese tipo en el pasado. No obstante, se insinúa que hay quienes no quieren que se negocie la paz con la guerrilla de las FARC (Implicatura general 2). El enunciado (2) además evoca la idea de «guerra», que ya se ha expresado en el enriquecimiento pragmático del estudio.

La desambiguación de la interjección mamola permite observar el matiz que le da al enunciado (2). Esta toma parte del sentido 2 de la RAE: [MAMOLA $2=$ interj. U. para expresar burla o negación]. Entonces se interpreta como [MAMOLA $3=$ interj. U. para expresar negación]. De este modo, el candidato Horacio Serpa se rehusó a que siguiera la guerra, lo que sugiere un apoyo al proceso de paz.

PLC, spot C: (3) Por la reparación de las víctimas del conflicto.

El enunciado sugiere que hay personas que han sido víctimas de un conflicto, es decir, la guerra armada entre la guerrilla de las FARC y el Gobierno Nacional (Implicatura general 3). Por ello, se insinúa que el gobierno debe garantizar la reparación a las víctimas de esa guerra.

PLC, spot C: (4) La paz para que vivas Mejor.

El enunciado implica que en Colombia no hay paz debido a una guerra entre la guerrilla de las FARC y el Gobierno Nacional (Implicatura general 4). Se infiere que la guerra ha provocado sufrimiento a la población civil por haber sido víctima de reclutamiento forzado, secuestro, extorsión, asesinato, despojo de bienes y tierras, lesiones permanentes al cuerpo, intimidación, etc. De este modo, se sugiere que, eliminando la guerra y sus efectos sobre la población civil, la gente puede vivir bien. Por lo tanto, considerando la Implicatura general 1, se insinúa que dichos efectos se pueden evitar, si se negocia la entrega de las armas y la integración a la vida civil de las FARC; con este enunciado, el PLC apoya las negociaciones de paz.

PLC, spot C: (5) Por una salida política negociable al conflicto. 
El enunciado sugiere que hay un conflicto armado en el país que se puede acabar a través de la negociación (Implicatura general 5), la que se propone en la Implicatura general 1. Por lo tanto, si se vota por el candidato Gustavo Moncayo, él seguirá apoyando esa negociación para llegar al final de la guerra. Esta última implicatura adquiere mayor importancia a partir del enriquecimiento pragmático que se le aplica al enunciado, ya que muestra un compromiso por la paz (mayor información en la introducción).

PLC, spot D: (6) En la clase de ayer nos dijeron que con lo que cuesta la guerra en un año se podrían entregar más de 80000 becas universitarias, construir más de 1000 colegios y escuelas y hacer más de 4000 km en vías y muchas cosas más.

El enunciado sugiere que la guerra es costosa en términos económicos, por lo cual, al gastar el presupuesto de la nación en esta hace que no se invierte en educación, vías y otras cosas.

PLC, spot D: (7) por eso se debe terminar, esto nos sacó del pueblo y se llevó la vida de tu tío Jaime.

El enunciado (7) se interpreta junto al enunciado (6), y así se resuelve la referencia de la frase «esto nos sacó del pueblo». En este caso, esto se refiere a la «guerra»; de otro lado, la desambiguación del sentido de llevar se adecua al sentido 22 de la RAE: [LLEVAR= prnl. Quitar algo a alguien, en general con violencia, o furtivamente]. Entonces, el enunciado sugiere que la guerra provoca el desplazamiento de las familias de sus residencias y, por lo tanto, muchas personas inocentes han muerto.

PLC, spot D: (8) (Martín, el niño): ¿Entonces la paz es que todos seamos felices? (El abuelo): Sí, Martín, la paz es para que todos vivamos mejor.

Este enunciado requiere resolver la referencia propuesta en todos, palabra que puede referirse a los colombianos. Así, se insinúa que la paz haría que los colombianos estuvieran tranquilos y, 
en consecuencia, felices. Entonces, se infiere que los colombianos no gozan de tranquilidad ni son felices.

Si (8) se interpreta a partir del contenido completo del spot D, se infiere que, si hay paz, la inversión de los recursos públicos se destinarían para la educación, la construcción de vías y otros gastos sociales. Con este tipo de inversiones, se mejorarían las condiciones de vida. Asimismo, se sugiere que, si se da la paz, no morirían más personas inocentes y las familias ya no tendrían que sufrir el desplazamiento forzado. Entonces, se insinúa que falta una mayor inversión económica en educación, vías y otros proyectos sociales.

PDA spot N: (9) Llegó el momento de la paz. Con esperanza vamos a construir un nuevo país sin víctimas, con tierra para los campesinos, con verdadera democracia.

En primer lugar, este enunciado insinúa la Implicatura general 1. Al mismo tiempo, con la frase con esperanza se sugiere que la paz no está asegurada. Luego, esto implica que la guerra ha generado víctimas, dentro de las cuales están los campesinos, quienes no son propietarios de las tierras que trabajan. De esta última idea, se infiere que el Estado colombia no actúa como una verdadera democracia, pues no permite que todos los ciudadanos tengan acceso a la propiedad sobre las tierras por igual.

PDA spot N: (10) En el Polo vamos por la paz.

El enunciado implica que el PDA está de acuerdo con la negociación de la paz con la guerrilla de las FARC.

PCC, spot H: (11) colombianos que no quieren la impunidad y piensan que con desarrollo habrá paz.

El enunciado sugiere que la impunidad se estaba negociando con la guerrilla de las FARC en La Habana, una de las sedes donde se llevaban a cabo las negociaciones de paz. Esa negociación conduce a perdonarle a ese grupo los delitos cometidos, pues ya ocurrió en el pasado cuando, el 8 
de marzo de 1990, la guerrilla M-19 recibió el indulto por sus delitos. De este modo, se insinúa que las FARC han cometido múltiples delitos contra la población civil, por lo que sería injusto que no tuvieran penas de encarcelamiento.

CD, spot Q: (12) Todos queremos la paz, pero queremos la paz seria, no firmada en un papel. Queremos la paz con hechos concretos, queremos la paz sin impunidad y sin terroristas en el Congreso de la República.

El enunciado insinúa la Implicatura general 1 y, a propósito de esta, sugiere que la negociación de la paz conduce a la impunidad de los hechos criminales cometidos por las FARC — pues ellos son terroristas_, lo que se daría a manera de amnistía y participación como congresistas de los principales cabecillas de esta guerrilla. Paralelamente, se insinúa que la firma de un acuerdo de paz no garantiza que se termine la guerra.

Los otros sentidos requieren la resolución de referencia de todos, término que se refiere a los colombianos en su conjunto. De este modo, se sugiere que los colombianos quieren una paz sin violencia, es decir, que las FARC dejen las acciones armadas y delictivas hacia la población civil y hacia las fuerzas armadas colombianas.

En conclusión, el enunciado implica que candidato Alfredo Ramos, del partido Centro Democrático, no permitiría que los terroristas de las FARC llegaran al Congreso de la República. En otras palabras, no firmaría los acuerdos de paz que se negociaban en La Habana.

CD, spot R: (13) Necesitamos obviamente la paz, pero no a cualquier precio, ni, de cualquier manera, una paz con justicia, una paz con desarme de los grupos terroristas, una paz sin participación política de los criminales de guerra y de lesa humanidad, una paz digna para el país, que realmente lleve a la reconciliación de los colombianos.

El enunciado insinúa la Implicatura general 1 y la Implicatura general 4. Además, sugiere que la paz que estaba negociando el Gobierno Nacional es una paz con injusticia, porque las FARC no irían a la cárcel por los crímenes que cometieron en el marco del conflicto armado. En 
este sentido, se implica que las FARC son un grupo terrorista y de criminales de guerra que han cometido delitos de lesa humanidad. Otro defecto de las negociaciones de paz, según se sugiere, es que las FARC conseguirían la participación en la política nacional. Frente a este escenario, el candidato insinúa que hay otras maneras de conseguir la paz, juzgando y encarcelando a los guerrilleros, quienes son criminales.

No obstante, el candidato reconoce explícitamente que «necesitamos la paz». El verbo necesitamos requiere una resolución de referencia porque supone un nosotros. En este caso, el nosotros se refiere a los colombianos, incluido el candidato. En conclusión, el candidato Alfredo Rangel no apoya las negociaciones del gobierno de Santos.

CR, spot J: (14) Algunos quieren llegar al Senado para atacar al presidente Santos. Yo, en cambio, quiero llegar al Congreso para trabajar por su salud, su educación, su vivienda y su progreso.

Este enunciado supone la Implicatura general 1, desde la cual insinúa que el presidente Santos tiene opositores políticos que son candidatos al Senado de la República, los cuales militan en el partido CD y se consideran uribistas. Así, como detractores de Santos, no aprobaron el proceso de paz del Gobierno con la guerrilla de las FARC. Por lo tanto, estos candidatos propusieron terminarían con las negociaciones de paz y lo harían desde el Congreso de la República; Por su parte, el candidato Dusan Pérez se negó a ser parte de esa pelea entre santistas y uribistas, al no oponerse ni apoyar los diálogos de paz.

OPIAC, spot T: (15) La Sierra Nevada y la Amazonia te invitan a hacer la paz con la naturaleza. Elige: aguas limpias, suelos vivos, semillas libres, la biodiversidad.

El enunciado requiere enriquecimiento pragmático respecto a los sustantivos la Sierra Nevada y la Amazonia. La Sierra Nevada alberga varias etnias, entre ellas los arhuacos, quienes, dentro de su cosmología, se consideran los «hermanos mayores». Según su cosmogonía, los hermanos mayores «son los encargados de cuidar y preservar el mundo, de velar porque el ciclo cósmico tenga un buen desarrollo para que las enfermedades no destruyan la vida de los hombres; para 
que las cosechas sean buenas» (Toda Colombia, 2017, § 3), y los demás serían los «hermanos menores». La Amazonia, por su parte, alberga 25 grupos étnicos (Corpoamazonia, s.f.). Al tratarse de pueblos indígenas, que viven una vida tradicional, estos se preocupan por el respeto del medio ambiente. También, al ser comunidades agrícolas, defienden la agricultura y luchan contra el tema del uso de semillas certificadas, impulsadas por el Gobierno y protegidas por los tratados de libre comercio firmados por Colombia. De hecho, la criminalización de campesinos por el uso indebido de semillas certificadas fue uno de los incidentes que provocó el Paro Agrario de 2013 y 2014, apoyado por la ciudadanía.

El enunciado sugiere que los colombianos no tienen una relación pacífica con la naturaleza, es decir, que no cuidan el agua, ni los suelos ni la biodiversidad. Así, la paz y la naturaleza implican la preservación del agua y de los suelos. También sugiere la protección de la biodiversidad y el uso de semillas libres, que no estén alteradas genéticamente, la cuales permitan continuar con la práctica tradicional de tomar parte de la cosecha como semilla.

\section{Discusión}

La conceptualización de la paz y la guerra se da principalmente de manera implícita, lo cual no es sorprendente en términos de la teoría de la relevancia, ya que los enunciados ofrecen al oyente una clave para la interpretación del sentido que el emisor desea comunicar. No obstante, también puede ser interpretado como una falta de compromiso de los candidatos y partidos de comunicar explícitamente su punto de vista sobre estos temas, uno que no dé lugar a dudas sobre cómo ellos los entienden. Pero, de otro lado, quizá tengan la intención de que cada oyente se forje su propia interpretación con la clave que se otorga (el mensaje codificado), lo que hace pertinente el mensaje a un grupo amplio de personas, que construyen el sentido según sus creencias.

Sin embargo, en cuanto al tema de la paz, estos hallazgos difieren de los de Botero (2018, pp. 16-17), quien afirmó que, en la contienda electoral legislativa de 2014, los partidos dieron pocas propuestas sustantivas y, en cambio, privilegiaron el tema de la negociación de la paz con las FARC. Este estudio nota que AICO no se refiere a la paz desde la perspectiva de las negociaciones de La Habana, mientras que la OPIAC no se ocupa ni de la paz ni de la guerra. Estos dos movimientos son organizaciones que buscan curules para el Congreso bajo la 
circunspección especial, dirigida a las minorías étnicas, especialmente las indígenas. Pese a que estas han quedado entre el fuego del Estado y el de las FARC, estos movimientos no se adhieren a los diálogos de paz, ni tampoco hacen referencia a la guerra. Quizá sea entendible en el caso de la OPIAC (Organización Nacional de Pueblos Indígenas de la Amazonia Colombiana), que alió a indígenas del Amazonas y de la Sierra Nevada de Santa Marta en el 2014, los cuales no han sufrido tanto la guerra como los pueblos indígenas de los departamentos de Nariño y Cauca, que son representados por el partido AICO (Autoridades Indígenas de Colombia).

De otro lado, los hallazgos de este estudio son semejantes a los encontrados en algunas investigaciones del macroproyecto de la Misión Observatorio Electoral (MOE, 2015) en cuanto a que el tema de la paz fue protagonico durante la campaña electoral al Congreso. Dicho macroproyecto se propuso revisar los medios nacionales y locales de mayor impacto que cubren el proceso electoral al Congreso y la Presidencia de la República en 2014 en los medios impresos, la televisión y la radio; aunque estos estudios no distinguen qué partidos políticos promovieron los temas explorados, identificaron los temas de campaña presente en los medios de comunicación anteriormente mencionados. La paz fue el tema más tratado en El Heraldo (de Barranquilla), el Nuevo Día (de Tolima), Ecos de Combeima, La Cariñosa de RCN (Martínez Heredia, 2015; González \& Perdomo, 2015). El de segunda importancia en El Tiempo, Caracol Radio (López, 2015). Y fue el tercer tema de mayor importancia en La Crónica del Quindío, en cuanto a la agenda de negociación de La Habana (Martinez, 2015, pág. 122).

No obstante, en otros medios, la presencia del tema de la paz fue mínimo. Ese fue el caso del Diario del Sur (de Nariño), el Diario la Opinión (de Norte de Santander) y Vanguardia Liberal (de Santander), donde aparecieron los temas de la «agenda de negociación» y «acuerdo humanitario» (Torres, 2015; Ferreira, 2015).

Así, el hecho de que partidos como el PLC, el CD y el PDA hubieran resaltado el tema de la paz no es sorprendente, dado que era el mayor asunto coyuntural del momento: los diálogos de paz del Gobierno Nacional con la guerrilla de las FARC para poner fin a la guerra interna de más de 50 años. Estos partidos representan dos visiones de país, el CD impuso la oposición a la paz tal como la plantea el gobierno de Santos, a través de la negociación. Del otro lado, el PLC y el PDA fueron los aliados de Santos al incluir en sus relatos una visión positiva sobre el fin del conflicto armado. 
Para Uribe y Rincón (2016), quienes estudiaron los anuncios televisivos de mayor resonancia y difusión de la campaña legislativa de 2014, una de esas posiciones apeló a un futuro en paz y más de lo mismo de Santos y, la otra, a un futuro-pasado que consiste en volver a los tiempos de AU como acto de restauración moral. Al mismo tiempo, afirman que esta campaña se caracterizó por usar la pedagogía del miedo con mensajes que se baten entre la apuesta por la guerra o por el ideal de paz. Paralelamente, en el corpus estudiado, la visión del fin de la guerra, en tanto la paz, desvía el asunto del surgimiento del conflicto, pues se insinúa que las FARC son un actor que caprichosamente inició la guerra y se les propone como el único grupo ilegal dentro del conflicto armado (Vázquez, 2014).

\section{Conclusiones}

El análisis evidenció que la conceptualización de la paz y la guerra se dio casi exclusivamente de manera implícita, lo que puede ser: 1. algo obvio en la comunicación según la teoría de la relevancia, ya que el enunciado es una clave para la interpretación del mensaje. Además, de un mensaje explícito pueden surgir también implicaturas. 2. Una falta de compromiso del candidato/partido de expresar explícitamente su visión del país, quizá con el fin de evitar entrar en debates sobre su posición. Lo anterior se debe que, según la teoría de la relevancia, el emisor expresa únicamente aquello que está dispuesto a comunicar. 3. Una estrategia para llegar a un número más amplio de votantes, pues un mensaje general o vago permite al votante guiar su interpretación hacia sus conocimientos o creencias más cercanas.

En estos casos se le da al televidente mayor responsabilidad sobre la interpretación, en la que se espera que sus conocimientos completen el sentido de lo comunicado y que dependen de la agenda pública. Entonces, si hay manipulación de la realidad a través de los mensajes de estos spots, esta depende de la agenda pública. Por eso, se propone estudiar — en otros trabajos — esta agenda como contexto cognitivo que favorece la interpretación y sus recursos de manipulación.

Según los hallazgos, Colombia vive una guerra protagonizada por las FARC, pero puede terminar si se culmina la negociación de paz entre el Gobierno Nacional y este grupo. Al respecto, las fuerzas políticas más poderosas en el territorio ( $\mathrm{PU}$ y $\mathrm{CD}$ ) impusieron dos interpretaciones de esta situación, mientras que $\mathrm{CR}$, AICO y OPIAC se mantuvieron neutrales - 
estos dos últimos, a pesar de que las comunidades que representa han sufrido directamente las consecuencias del conflicto armado-. Quienes estaban a favor de la negociación (PU, PLC, PDA), esperaban que la guerra terminara. Los sectores que están en contra advirtieron que se estaba negociando la impunidad de los guerrilleros frente a los delitos que cometieron, lo que no consideraban justo. El CD y el PCC se oponía a que los guerrilleros no pagasen penas de cárcel por los delitos de lesa humanidad que cometieron, pues consideraban que ellos, en cambio, recibirían curules en el Congreso.

$\mathrm{CR}$, por su parte, tan solo advirtió que había polarización debido a las posiciones de santistas y de uribistas. Mientras tanto, la OPIAC conceptualiza la paz en términos ambientales, catalogando la paz como un estado de armonía con la naturaleza.

\section{Referencias bibliográficas}

1. Alberto Castilla Senador. (25 de febrero de 2014). Alberto Castilla, candidato al Senado 20142018. [Archivo de video]. Recuperado de https://www.youtube.com/watch?v=70OHVAxiki8

2. Botero, F. (2018). Competencia desde la derecha. Las elecciones nacionales del 2014 y el sistema de partidos. En F. Botero, M. García Sánchéz, \& L. Wills-Otero (Eds.), Polarización y posconflicto: las elecciones nacionales y locales en Colombia, 2014-2017 (págs. 11-28). Bogotá: Universidad de los Andes.

3. Botero, F., García Sánchéz, M., \& Wills-Otero, L. (2018). Polarización y posconflicto: las elecciones nacionales y locales en Colombia, 2014-2017. Bogotá: Universidad de los Andes.

4. Campaña Carlosgalan. (26 de febrero de 2014). Carlos Fernando Galán habla sobre el congreso. [Archivo de video]. Recuperado de https://www.youtube.com/watch?v=o8PnIqllAC4 5. Casetti, F., \& di Chio, F. (2007). Cómo analizar un film. Barcelona: Paidós.

6. Centro Democrático Comunidad Oficial. (31 de enero de 2014). Alfredo Ramos-Nuestra visión de Paz: Centro Democrático [Archivo de video]. Recuperado de https://www.youtube.com/watch?v=UZeOD8NDD_I

7. Centro Democrático Comunidad Oficial. (31 de enero de 2014). Alfredo Rangel-Nuestra visión de Paz: Centro Democrático [Archivo de video]. Recuperado de https://www.youtube.com/watch?v=yOcy065xAgs 
8. Centro Democrático Comunidad Oficial. (30 de enero de 2014). María del Rosario Guerra¿Por qué los candidatos del CD quieren llegar al Congreso? [Archivo de video]. Recuperado de https://www.youtube.com/watch?v=h_0Wh6Ozolg

9. Colombia.com. (2014). Elecciones legislativas 2014: resultados de elecciones legislativas.

Recuperado

https://www.colombia.com/elecciones/2014/legislativas/resultados/electorales.aspx ?C=SE

10. Contamos con Vos. (24 de febrero de 2014). Iván Cepeda Senado 10 [Archivo de video]. Recuperado de https://www.youtube.com/watch?v=wnAcd_2ZBuI

11. Contreras, J. (2006). El encanto audiovisual en la política: el spot político-electoral televisivo. Recuperado de http://conocimientoabierto.flacso.edu.mx/tesis/120

12. Corpoamazonia. (s.f.). Etnias y culturas. Recuperado de http://www.corpoamazonia.gov.co/region/Etnias.htm

13. De Aragón, A. E. (2011). Cómo ganar el corazón y la mente de los votantes: estrategias y tácticas para una campaña electoral exitosa. Bloomington, (Estados Unidos de América): Palibrio.

14. El Espectador. (28 de enero de 2014). Comerciales polémicos de campañas políticas: Luis Emilio Sierra dArchivo de video]. Recuperado de https://www.youtube.com/watch?v=sHcD0kzEwIs

15. El Espectador. (28 de enero de 2014). Comerciales polémicos de campañas políticas: Nora García Burgos/Agro Colombiano [Archivo de video]. Recuperado de https://www.youtube.com/watch?v=gMYwLc3dipk

16. Ferreira, J. (2015). Cubrimiento de primera vuelta elecciones presidenciales 2014 en el departamento de Santander desde una mirada a los medios impresos Vanguardia Liberal y El Frente. En MOE, Elecciones y medios de comunicación: un zoom a las mujeres en la agenda política, (Tomo 2), (pp. 105-111). Bogotá: Grupo DAO Digital Limitada.

17. García Beaudoux, V., D’Adamo, O. \& Slavinsky. (2005). Comunicación política y campañas electorales: estrategias en elecciones presidenciales. Barcelona: Gedisa.

18. Gerlein, R. (28 de febrero de 2014). Comercial Senador Roberto Gerlein. Recuperado de https://www.youtube.com/watch?v=ltrPCdVg5_s 
19. Germán Fernández Candidato al Senado. (23 de febrero de 2014). Dr. Germán Fernández. Candidato al Senado Polo 16 [Archivo de video]. Recuperado de https://www.youtube.com/watch?v=kMLoZ69xHe0

20. González, R., \& Perdomo, C. (2015). Resultados del seguimiento a los medios Nuevo Día, La Cariñosa RCN, Ecos del Combeima en elecciones presidenciales. MOE, Elecciones y medios de comunicación: un zoom a las mujeres en la agenda política, (Tomo 2), (pp. 113-120). Bogotá: Grupo DAO Digital Limitada.

21. Hernández Bayter, H. (2015). El debate Santos-Zuluaga, el uso de formas y secuencias recurrentes como mecanismos lingüístico-discursivos. Soprag, vol. 3, no. 2. 174-203. DOI 10.1515 .

22. Lafuente, Javier; Palomino, Sally; Marcos, Ana. (2016). Colombia: el camino a la paz. Recuperado de https://elpais.com/especiales/2016/proceso-de-paz-en-colombia/ 23. López Maya, A. (01 de marzo de 2014). Alexander López Maya candidato al Senado Polo \# 2 [Archivo de video]. Recuperado de https://www.youtube.com/watch?v=O2-biIC78yw

24. López Obregón, C. E. (20 de abril de 2014). Una Clara propuesta para el Agro. Clara López presidenta [Archivo de video]. Recuperado de https://www.youtube.com/watch?v=myUMwX5a1mI

25. López, R. A. (2015). Análisis sobre el cubrimiento de las elecciones presidenciales en Colombia 2014 por los medios de comunicación el tiempo, eltiempo.com y lasillavacia.com. En MOE, Elecciones y medios de comunicación: un zoom a las mujeres en la agenda política, (Tomo 2), (pp. 50-61). Bogotá: Grupo DAO Digital Limitada.

26. Luzardo Ocampo, A. M. (8 de mayo de 2014). Campaña Ati Quigua Senado 2014 [Archivo de video]. Recuperado de https://www.youtube.com/watch?v=qUD6lcT9_O4

27. Martínez Heredia, K. M. (2015). Una mirada reflexiva a las elecciones de Congreso y Presidencia 2014 en el Departamento del Atlantico desde el seguimiendo al mass media impreso El Heraldo. MOE, Elecciones y medios de comunicación: un zoom a las mujeres en la agenda política, (Tomo 2), (pp. 39-49). Bogotá: Grupo DAO Digital Limitada.

28. Misión de Observación Electoral. (2015). Elecciones y medios de comunicación: un zoom a las mujeres en la agenda política, (Tomo 2). Bogotá: Grupo DAO Digital Limitada. 
29. Pachón, M. (2014). Las elecciones de Colombia 2014: el tortuoso camino para darle un segundo tiempo al proceso de paz. Iberoameticana, No XIV, 55, 181-187.

30. Partido Conservador Colombiano. (18 de febrero de 2014). Comercial Partido Conservador Colombiano [Archivo de video]. Recuperado de https://www.youtube.com/watch?v=1zkY_a18fI0

31. Partido Liberal Colombiano. (6 de febrero de 2014). Guillermo García Realpe L20 Senado [Archivo de video]. Recuperado de https://www.youtube.com/watch?v=Tezwe1nGeaE 32. Partido Liberal Colombiano. (19 de febrero de 2014). Horacio Serpa Senado 1 [Archivo de video]. Recuperado de https://www.youtube.com/watch?v=zYqO0OT82b4 33. Partido Liberal Colombiano. (3 de marzo de 2014). La Paz Para Que Vivas Mejor [Archivo de video]. Recuperado de https://www.youtube.com/watch?v=2fRcIp8YUmo

34. Partido Liberal Colombiano. (19 de febrero de 2014). Vota Partido Liberal Colombiano, Para que Vivas Mejor [Archivo de video]. Recuperado de https://www.youtube.com/watch?v=qAeVqSwHdYo

35. Pedraza, J. H. (17 de enero de 2014). El \#BuenCamino con Jorge Hernando Pedraza [Archivo de video]. Recuperado de https://www.youtube.com/watch?v=G9CmQDLmOwc 36. Pons Bordería, S. (2004). Conceptos y aplicaciones de la Teoría de la Relevancia. Madrid: Arco Libros.

37. Richard, E. (29 de septiembre de 2015). Elecciones presidenciales en Colombia en 2014: construcción de relatos de paz. Revista Zero, 33. ISSN 2344-8431.

38. Rivas, J. M., \& Roll, D. (2016). Los acuerdos de paz: ¿tema central de los partidos en la campaña presidencial de 2014 en Colombia. Ciencia Política, vol. 11, no. 21, 365-396. doi: https://doi.org/10.15446/cp.v11n21.60298

39. Toda Colombia. (14 de 12 de 2017). Arhuaco-Ika. Toda Colombia: la cara amable de Colombia. Recuperado de http://www.todacolombia.com/etnias-de-colombia/gruposindigenas/arhuaco.html

40. Torres, F. (2015). El comportamiento del Diario del Sur. MOE, Elecciones y medios de comunicación: un zoom a las mujeres en la agenda política, (Tomo 2) (pp. 90-98). Bogotá: Grupo DAO Digital Limitada. 
41. Uribe, C., \& Rincón, O. (2016). Un país, muchos candidatos y un sólo ganador: campaña electoral Colombia 2014 y spots televisivos. En O. Leyva, (Ed.), El spot político en América Latina: enfoques, métodos y perspectivas (pp. 193-231). Puerto Vallarta (México): Universidad de Guadalajara.

42. Vázquez, U. (2014). El conflicto colombiano: incidencia de la política de seguridad democrática uribista en la seguridad humana. Nómadas. Revista Crítica de Ciencias Sociales y Políticas, 44(4), 163-175. http://dx.doi.org/10.5209/rev_NOMA.2014.v44.n4.49299

43. Vilches, L., del Río, O., Simelio, N., Soler, P. \& Velázquez, T. (2011). La investigación en comunicación: métodos y técnicas en la era digital. Barcelona: Gedisa.

44. Wilson, D. \& Matsui, T. (2012). Recent approaches to bridging; truth, coherence, relevance. En D. Wilson, \& D. Sperber, (Eds.), Meaning and Relevance (pp. 187-209). New York: Cambridge University Press.

45. Wilson, D. \& Sperber, D. (2005). Teoria da relevância. Liguagem em (Dis)curso, v. 5, n. esp., 221-268.

46. Wilson, D. \& Sperber, D. (2012). Meaning and relevance. United Kingdom: Cambridge. 